\title{
Some notes on Second Countablility in Frames
}

\author{
Mary Elizabeth Antony ${ }^{1}$, Sabna.K.S. ${ }^{2}$,Dr.Mangalambal N.R. ${ }^{3}$ \\ ${ }^{I}$ (Mathematics Dept., Mar Athanasius College/ Mahatma Gandhi University, India) \\ ${ }^{2}$ (Mathematics Department, K.K.T.M College/Calicut University, India) \\ ${ }^{3}$ (Mathematics Department, St.Joseph'sCollege/Calicut University, India)
}

Abstract: In this paper we have tried to develop second countability in frames parallel to that in classical topology.

Keywords: $B_{L}^{2}$ frame, Dense Sublocale, L-base, Locales, Lower frame.

\section{Introduction}

With the work of Marshall Stone on the topological representation of Boolean algebras and distributive lattices, the connection between topology and lattice theory began to be explored.

As a dual notion of category of frames we have the category of locales. The category of pointless topological space called locales is an extension of ordinary topological space .

In locales the concept of points gets replaced with "opens". Thus the introduction to the concepts of pointless topology was laid. Most concepts of point set topology like separation axioms ,compactification ,etc... have already be defined into the contexts of locales and proved the analogues theorems.

Here we have tried to define second countability and separability in the contexts of locale theory.

2.1 Frame [1] : A poset $(\mathrm{A}, \leq)$ is a frame if and only if

\section{Preliminaries}

i)Every subset has a join

ii)every finite subset has a meet

iii)binary meets distribute over joins :

$\mathrm{x} \wedge \mathrm{V} \mathrm{Y}=\mathrm{V}\{\mathrm{x} \wedge \mathrm{y}: \mathrm{y} \epsilon \mathrm{Y}\} \quad$ (frame distributivity)

2.2 Boolean Algebra[2]:A Boolean algebra is a distributive lattice A equipped with an additional unary operation $\rceil: \mathrm{A} \rightarrow \mathrm{A}$ such that $\rceil \mathrm{a}$ is a complement of $\mathrm{a}$ i.e $\rceil \mathrm{a} \wedge \mathrm{a}=0$ and $\rceil \mathrm{a} \vee \mathrm{a}=1$.

2.3 Heyting Algebra[2] : A lattice A is said to be a Heyting algebra ,if for each pair of elements (a,b)there exists an element $(a \rightarrow b)$ such that $c \leq(a \rightarrow b)$ iff $c \wedge a \leq b$.

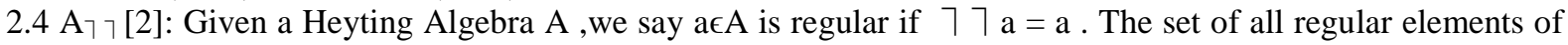
$\mathrm{A}$ with its induced order, is denoted $\mathrm{A}_{\urcorner} 7$.

2.5 Minimal Elements[3] :Let $\mathrm{A}$ be a poset. An element $\mathrm{x} \in \mathrm{A}$ is said to be a minimal element iff $\mathrm{c} \leq \mathrm{a}$ implies $\mathrm{c}=\mathrm{a}$.

2.6 Frame homomorphism[1] : A function between two frames is a frame homomorphism iff it preserves all joins and finite meets.

2.7 Frm[2]: this is the category of frames whose objects are complete lattices satisfying the infinite distributive law, and whose morphisms are functions preserving the finite meets and arbitrary joins

$2.8 \mathrm{Loc}[2]$ : It is the dual of Frm. And the frames are now referred to as Locales.

2.9 Sublocale[3]:A subset $\mathrm{S} \subseteq \mathrm{L}$ is a sublocale if

i)it is closed under all meets

ii)for every $s \in S$ and every $x \in L, x \rightarrow s \in S$.

With the help of these preliminaries, we have tried to develop some definitions and a few theorems corresponding to it.

\section{Second countability}

3.1 L-base

Definition : A collection $B_{\mathrm{L}}$ of elements of a locale $\mathrm{L}$ is said to be $\mathrm{L}$-base for the locale $\mathrm{L}$ if for every $\mathrm{a}(\neq 0) \in \mathrm{L}$ ,there exists a non empty sub-collection $\left\{b_{i}: b_{i} \in B, i \in \Delta\right\}$ such that

$$
V_{i \in \Delta} b_{i} \leq a \text { and } \mathrm{V}\left\{b_{i}: b_{i} \in \mathrm{B}\right\}=1 \text {. }
$$

\subsubsection{Examples}

3.1.1.1. In the background of frames the class $B_{\mathrm{L}}$ of all singletons form a base for Discrete topology on $\mathrm{X}$ 3.1.1.2.For a frame 


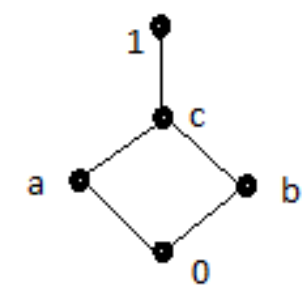

$B_{\mathrm{L}}=\{\mathrm{a}, \mathrm{b}\}$

3.1.1.3. In the background of frames the collection $B_{\mathrm{L}}$ of compact sets forms a base for locally compact spaces.

3.1.1.4.In the frame of Upper closed sets of finite (say $1,2,3, \ldots . . \mathrm{N}$ ) list of bits 0,1 ,the collection

$B_{\mathrm{L}}=\{\uparrow 1: 1$ is a list of length $\mathrm{N}\}$ form L-base

3.1.2 Remark 1

If $B_{\mathrm{L}}$ is a L-base for the frame (locale) L iff $\forall \mathrm{p} \in \mathrm{L}$ there exists some $\mathrm{b}_{\mathrm{p}} \in B_{\mathrm{L}}$ such that $\mathrm{b}_{\mathrm{p}} \leq \mathrm{p}$.

Proof

Let $B_{\mathrm{L}}$ be a L-base for $\mathrm{L}$ then $\forall \mathrm{p} \in \mathrm{L}$ there exists some $\left\{\mathrm{b}_{\mathrm{i}}: \mathrm{i} \in \Delta\right\}$ such that

$$
V b_{i} \leq \mathrm{p}
$$

$$
\exists b_{\mathrm{p}} \leq V b_{i} \leq \mathrm{p}, \text { where } b_{\mathrm{p}} \in\left\{\mathrm{b}_{\mathrm{i}}: \mathrm{i} \in \Delta\right\} .
$$

Now suppose $\forall \mathrm{p} \in \mathrm{L}$ there exists $\mathrm{b}_{\mathrm{p}} \in B_{\mathrm{L}}$ such that $\mathrm{b}_{\mathrm{p}} \leq \mathrm{p}$. Let $\mathrm{x} \in \downarrow_{\mathrm{p}}$ then $\forall \mathrm{x} \in \downarrow_{\mathrm{p}}$, by our assumption there exists $\mathrm{b}_{\mathrm{x}} \in B_{\mathrm{L}}$ such that $\mathrm{b}_{\mathrm{x}} \leq \mathrm{x} \leq \mathrm{p}$

Therefore $V b_{x} \leq \mathrm{p} \forall \mathrm{x} \in \downarrow_{\mathrm{p}}$.

Thus $B_{\mathrm{L}}$ is a base for the given locale $\mathrm{L}$

3.1.3 Remark 2

Let $B_{\mathrm{L}}$ be a $\mathrm{L}$ base for some frame(locale) $\mathrm{L} B_{\mathrm{L}} *$ be a class containing $B_{\mathrm{L}}$ ie,

$B_{\mathrm{L}} \subseteq B_{\mathrm{L}} *$ then $B_{\mathrm{L}} *$ is also a $\mathrm{L}$-base for $\mathrm{L}$.

Proof

Let $\mathrm{p} \in \mathrm{L}$ then by remark 1 we know that there exists $\mathrm{b}_{\mathrm{p}} \in B_{\mathrm{L}}$ such that $\mathrm{b}_{\mathrm{p}} \leq \mathrm{p}$. Since

$B_{\mathrm{L}} \subseteq B_{\mathrm{L}}{ }^{*}, \mathrm{~b}_{\mathrm{p}} \in B_{\mathrm{L}} *$ implies $\mathrm{b}_{\mathrm{p}} \leq \mathrm{p}$ for some $\mathrm{b}_{\mathrm{p}} \in B_{\mathrm{L}}{ }^{*}$. So by Remark 1 we have $B_{\mathrm{L}}{ }^{*}$ is also a

$\mathrm{L}$ - base for $\mathrm{L}$.

3.2 Theorem

Let $B_{\mathrm{L}}$ be a collection of elements of $\mathrm{L}$. Then $B_{\mathrm{L}}$ is a $\mathrm{L}$-base for $\mathrm{L}$ if for any $\mathrm{b}_{1}, \mathrm{~b}_{2} \in B_{\mathrm{L}}$ there exists some $\mathrm{b}_{3} \in B_{\mathrm{L}}$ such that $\mathrm{b}_{3} \leq \mathrm{b}_{1} \wedge \mathrm{b}_{2}$.

Proof

Let $B_{\mathrm{L}}$ be a L-base then for $\mathrm{b}_{1}, \mathrm{~b}_{2} \in B_{\mathrm{L}}, \mathrm{b}_{1} \wedge \mathrm{b}_{2} \in \mathrm{L}$ ( $\mathrm{L}$ being a frame).Then ,by the definition of base there exists $b_{3} \in B_{L}$ such that $b_{3} \leq b_{1} \wedge b_{2}$.

3.2 Lower frame

3.2.1Definition :Let $\mathrm{L}$ be a frame with base $B_{\mathrm{L}}$ and let $\mathrm{L}^{*}$ be a frame with base $B_{\mathrm{L}^{*}} *$. Then we say that $\mathrm{L}$ is a lower frame to $\mathrm{L}^{*}$, denoted as $\mathrm{L} \prec_{B} \quad \mathrm{~L}^{*}$ if for every $\mathrm{b} \in B_{\mathrm{L}}$

$\mathrm{b}=\mathrm{V}\left\{\mathrm{b} * \mathrm{~b} * \in B_{\mathrm{L}^{*}} *\right\}$.

3.2.1 Example : $\mathrm{R}$ with usual topology is a lower frame to $\mathrm{R}$ with upper limit topology.

3.3 2-countability

3.3.1 Definiton

A frame $\mathrm{L}$ is said to be a $B_{\mathrm{L}}{ }^{2}$ frame if it has a countable L- base. And this property is called 2countability.

\subsubsection{Example}

The frame of Upperclosed sets of finite list of bits 0and 1 is second countable.

3.3.3 Theorem

2-countability is hereditary.

Proof

Let $\mathrm{L}$ be $B_{\mathrm{L}}{ }^{2}$ frame. Let $\mathrm{h}: \mathrm{L} \rightarrow \mathrm{M}$ be a sublocale.

Let $B=\left\{b_{1}, b 2, \ldots\right\}$ be a countable base for $L$.

Then we will show that $\{h(b 1), h(b 2),,$,$\} is a countable base for M$

Let $c \in M$,then since $h$ is onto there exists $a \in L$ such that $h(a)=c$

$\mathrm{a} \in \mathrm{L} \Rightarrow \mathrm{V} \mathrm{b}_{\mathrm{i}} \leq \mathrm{a} \Rightarrow \mathrm{h}\left(\mathrm{Vb}_{\mathrm{i}}\right) \leq \mathrm{h}(\mathrm{a})$

$$
\Rightarrow \mathrm{Vh}\left(\mathrm{b}_{\mathrm{i}}\right) \leq \mathrm{h}(\mathrm{a})=\mathrm{c}
$$




\subsection{Lindeloff}

If every cover $U=\left\{\mathrm{U}_{\alpha}: \alpha \in \mathrm{A}\right\}$ of a frame $\mathrm{L}$ has a countable sub cover then $\mathrm{L}$ is said to be Lindeloff.

3.4.1 Theorem

Every 2- countable frame $\mathrm{L}$ is Lindeloff.

Proof

Let $B_{\mathrm{L}}$ be a countable $\mathrm{L}$ - base for $\mathrm{L}$.Suppose $U$ is any cover of $\mathrm{L}$.For each $\mathrm{U} \in U$ there is

some $\mathrm{b}_{1, \mathrm{U}} \in B_{\mathrm{L}}$ such that $\mathrm{b}_{1, \mathrm{U}} \leq \mathrm{U}$.

Now $B^{\prime}=\left\{\mathrm{b}_{1, \mathrm{U}}: \mathrm{U} \in U\right\}$ is a countable set , since $B^{\prime} \subseteq B_{\mathrm{L}}$, say $B^{\prime}{ }^{\prime}=\left\{\mathrm{b}_{\mathrm{i}, \mathrm{U} 1}, \mathrm{~b}_{2, \mathrm{U} 2,} \mathrm{~b}_{3, \mathrm{U}}\right.$,

Since $B$ is a $L$ base we know that $1=V\left\{\mathrm{~b}: \mathrm{b} \in B_{\mathrm{L}}\right\}$ Then $\mathrm{U}_{1} U_{2}, \ldots$ is a countable sub-cover from $U$.

3.4.2 Remark

If $\mathrm{L}$ is $B_{\mathrm{L}}{ }^{2}$ then clearly the set of all minimal elements is countable.

3.4.3 Proposition [2]

A $\neg$ is a Boolean Algebra

For Proof see [2].

3.4.4 Lemma [2]

Every locale has a smallest dense sublocale, A $\neg$ -

For Proof see [2]

3.4.5 Dense sublocale[3]

A sublocale $S$ of a locale $A$ is said to be dense if $\bar{S}=\uparrow(\Lambda S)=A$.Or equivalently $S$ is dense iff it

$0_{\mathrm{A}} \in \mathrm{S}$

\section{Separability}

As in classical topology, a frame is said to be separable if it has a countable dense sublocale.

\subsection{Theroem}

Proof

Every $B_{\mathrm{L}}{ }^{2}$ frame is separable.

From the above lemma we know that every locale A has a smallest dense sublocale $A$ ר

So if we can prove that $A \neg$ is countable then we are through.Let $B_{\mathrm{L}}=\left\{\mathrm{b}_{1}, \mathrm{~b}_{2}, \mathrm{~b}_{3}, \ldots ..\right\}$ be a base for the locale A..

If we can prove that $A \neg \neg \subseteq B_{\mathrm{L}}$, then we are done.

Now let $\mathrm{a} \in \mathrm{A} \neg$ and $\mathrm{a} \notin B_{\mathrm{L}}$.

$\neg \mathrm{b}_{\mathrm{i}} \in \mathrm{A} \neg \neg \forall \mathrm{b}_{\mathrm{i}}$

$\mathrm{b}_{1} \$ \mathrm{a} \wedge \neg \mathrm{b}_{1}$

$\mathrm{b}_{2} \nsubseteq \mathrm{a} \wedge \neg \mathrm{b}_{2}$

i..e, in general , $\mathrm{b}_{\mathrm{i}} \sharp \mathrm{a} \wedge \neg \mathrm{b}_{\mathrm{i}}$

$\therefore$ There exist no element $\mathrm{b}_{\mathrm{i}} \in B_{\mathrm{L}}$ such that $\mathrm{b}_{\mathrm{i}} \leq \Lambda\left\{\mathrm{a} \wedge \neg \mathrm{b}_{1}, \mathrm{a} \wedge \mathrm{b}_{2}, \mathrm{a} \wedge \neg \mathrm{b}_{3} \ldots \ldots \ldots \ldots . . . ..\right\}$. But this is not possible since $B_{\mathrm{L}}$ is a base for the sublocale $A \neg$ ᄀ

Thus a $\in B_{\mathrm{L}}$. Here a was an arbitrary element from $A \neg \neg$, hence $A \neg \neg \subseteq B_{\mathrm{L}}$.

A being $B_{\mathrm{L}}{ }^{2}$, we have $B_{\mathrm{L}}$ to be countable , hence A $\mathrm{h}$ is countable .

Thus A is separable.

4.2 Lemma

Let $\mathrm{h}: \mathrm{A} \rightarrow \mathrm{B}$ be a frame homomorphism. If $\mathrm{a}$ is a regular element of $\mathrm{A}$, then $\mathrm{h}(\mathrm{a})$ is a regular element in $\mathrm{B}$

Proof

Since $\mathrm{a}$ is a regular element in A,we have $\rceil 7 \mathrm{a}=\mathrm{a}$

Ie, $\mathrm{a} \vee\urcorner \mathrm{a}=1$ and $\mathrm{a} \Lambda\rceil \mathrm{a}=0$

Since $\mathrm{h}$ is a frame homomorphism we have

$\mathrm{h}(\mathrm{a} \Lambda\urcorner \mathrm{a})=\mathrm{h}(0)=0_{\mathrm{B}}$ and $\left.\mathrm{h}(\mathrm{a} \vee\urcorner \mathrm{a}\right)=\mathrm{h}(1)=1_{\mathrm{B}}$

ie, $h(a) \Lambda h(\urcorner a)=0_{B}$ and $\left.h(a) \vee h(\urcorner a\right)=1_{B}$

ie, $h(\rceil a)=\urcorner h(a)$

hence $\rceil 7 \mathrm{~h}(\mathrm{a})=\urcorner(\rceil \mathrm{h}(\mathrm{a}))=\urcorner \mathrm{h}(\urcorner \mathrm{a})=\mathrm{h}(\rceil 7 \mathrm{a})=\mathrm{h}(\mathrm{a})$

Therefore, $\mathrm{h}(\mathrm{a})$ is a regular element in B.

4.3Theorem

Homomorphic image of a separable space is separable

Proof

Let $\mathrm{A}$ be separable and let $\mathrm{h}: \mathrm{A} \rightarrow \mathrm{B}$ is a frame homomorphism. 
Since $A$ is s separable, $\left.A_{7}\right\urcorner$ is a countable dense sublocale of A. Now consider $\left.h(A\urcorner 7\right)$.Clearly this is a countable and dense. Hence $\mathrm{h}(\mathrm{a})$ is separable.

\section{Conclusion}

Frame theory finds its applications in computer science where the domain theory provides a mathematical foundation for semantics of programming languages. Here we have developed the concepts of second countability and separability in locale theory. Further study can be done in developing an equivalent theory for local base and first countability

\section{Books}

\section{References}

[1] Steven Vickers,Topology via logic(Cambridge Tracts in Theoretical Computer Science,Cambridge University Press1989)

[2] Peter.T.Johnstone,Stone Spaces(Cambridge University Press,1982)

[3] Jorge Picardo, Ales Pultr ,Frames and Locales - Topology without points(springer Basel AG 2012) 